Background While systemic lupus erythematosus (SLE) disproportionately affects minority women of child-bearing age, $1520 \%$ of all patients with SLE are diagnosed as children. Studies have shown that patients with SLE perceive their quality of life as poorer than the general public due to the impact of the disease on aspects of their physical, social, and psychological function. This study compares the health-related quality of life (HRQOL) in childhood-onset SLE (cSLE), defined as diagnosed prior to age 18 , to HRQOL in adult-onset SLE (aSLE).

Methods Data was collected as part of an ongoing longitudinal SLE registry at MUSC, including demographics, clinical disease manifestations and patient-reported responses to the Short Form-36 (SF-36) v2 questionnaire. For this study, the initial SF-36 v2 questionnaire completed at time of registry enrollment after age 18 was utilized, excluding patients with less than 4 ACR Classification Criteria for SLE and patients missing SF-36 v2 data. Scores were analyzed across four physical health and four mental health domains. SF-36 v2 mean scores were compared between cSLE patients and aSLE patients.

Results The mean normalized scores for all four SF-36 v2 physical health domains were higher for cSLE patients compared to aSLE patients as seen in table 1 . There was statistical significance found in two domains of physical health: physical functioning $(52.1 \pm 6.1 \mathrm{cSLE}$ vs $38.8 \pm 12.4 \mathrm{aSLE}, \mathrm{p}=0.002)$ and role physical $(50.1 \pm 7.1 \mathrm{cSLE}$ vs $38.5 \pm 12.5 \mathrm{aSLE}$, $\mathrm{p}=0.007)$. The mean normalized scores for all four SF-36 v2 mental health domains were also higher for cSLE patients compared to aSLE patients, but no statistical significance was found.

Conclusions Despite the association among patients with SLE between childhood-onset and a more severe disease course, our findings showed an overall higher health related quality of life within the cohort. These findings suggest that having been diagnosed at an earlier age and having lived most of their life with a systemic autoimmune disease may contribute to the higher quality of life reported by patients with cSLE.

Funding Source(s): This work has been supported by funding from the Rheumatology Research Foundation 2016 Medical Student Research Preceptorship (CLK) and the National Institutes of Health: Medical University of SC Clinical and Translational Science Award UL1 RR029882 and National Institute of Arthritis and Musculoskeletal and Skin Diseases Award P60 AR062755 (GSG, JCO, DLK) and K24 AR068406 (DLK), and the VA Medical Service at the Ralph $\mathrm{H}$. Johnson VA Medical Center (GSG, and JCO).

\section{CHARACTERIZATION OF A SINGLE CENTER SYSTEMIC LUPUS ERYTHEMATOSUS COHORT ACCORDING TO COMPLEMENT LEVELS}

${ }^{1}$ Sheyla Leal Castro*, ${ }^{2}$ Luis 0 Roa Perez, ${ }^{3}$ Yesid J Portilla, ${ }^{2}$ Adriana Seewald, ${ }^{3}$ Jaime A Betancourt, ${ }^{4}$ Sandy Sapag Duran, ${ }^{5}$ Diana Dubinsky. ${ }^{1}$ University of Buenes Aires; ${ }^{2}$ Hospital de Clínicas Jose de San Martin; ${ }^{3}$ University of Buenos Aires; ${ }^{4}$ Hospital de Clínicas Jose de San Martpin; ${ }^{5}$ Sanatorio Guemes

\subsection{6/lupus-2019-|sm.155}

Background Systemic Lupus Erythematosus (SLE) is an autoimmune disease in which inflammation is mediated by complement activation by immune complex deposit, leading to damage. Consumption of C3 and C4 complement fragments is accepted as a disease activity marker.

\begin{tabular}{|c|c|c|c|c|c|c|}
\hline & $\begin{array}{l}\text { (G1) } \\
\text { Normal C3 and C4 }\end{array}$ & $\begin{array}{l}\text { (G2) } \\
\text { Low levels C3 and C4 }\end{array}$ & $\begin{array}{l}\text { (G3) } \\
\text { Low levels C4 }\end{array}$ & $\begin{array}{l}\text { (G4) } \\
\text { Variable levels C3 and/or C4 }\end{array}$ & $\begin{array}{l}\text { Total } \\
\text { n } 149\end{array}$ & $P$ (value) \\
\hline Age at diagnosis & $32,1(13,9)$ & $29,5(10,9)$ & $28,5(15,4)$ & $29,5(13,6)$ & $30,6(13,3)$ & 0004 \\
\hline \multicolumn{7}{|l|}{ ISD) } \\
\hline $\begin{array}{l}\text { Disease duration } \\
\text { (mean yrs) }\end{array}$ & 10,5 & 7,8 & 13,07 & 8,7 & 9,7 & 0002 \\
\hline Female (\%) & $98,5 \%$ & $94,1 \%$ & 92,9 & 90,9 & 95,3 & 0001 \\
\hline $\begin{array}{l}\text { SLEDAl } \\
\text { (mean/SD) }\end{array}$ & $5,1(4,5)$ & $9.2(4,75)$ & $8,07(4)$ & $7,9(5,4)$ & $6,9(5.0)$ & 0001 \\
\hline SLICC (average) & 1,01 & 0,5 & 1,3 & 0,6 & 0.8 & 0031 \\
\hline Leukopenia (\%) & 27,9 & 29,4 & 21,4 & 27,3 & 27,5 & 0.876 \\
\hline Lymphopenia (\%) & 27,9 & 32,9 & 21,4 & 30.3 & 28,9 & 0769 \\
\hline Hemolytic anemia (\%) & 17,6 & 5,9 & 28,6 & 6,1 & 13,4 & 0196 \\
\hline Hypergamma (\%) & 30,9 & 30,3 & 50 & 44.1 & 35,6 & 0.211 \\
\hline LN (\%) & 36,8 & 45,5 & 78,6 & 35,3 & 42.3 & 0,0002 \\
\hline Overlap (\%) & 11,8 & 15,2 & 14,3 & 11,8 & 12,8 & 0,55 \\
\hline DNAds (\%) & 26,3 & 39,4 & 50 & 26,5 & 42,3 & 0011 \\
\hline Sm (\%) & 14,5 & 30,3 & 14,3 & 38,2 & 35,4 & 0,0001 \\
\hline RNP (\%) & 22,1 & 33,3 & 7,1 & 44,1 & 42,4 & 0003 \\
\hline Ro (\%) & 35,3 & 48,5 & 35,7 & 50 & 55,9 & 0,32 \\
\hline La (\%) & 16,2 & 15,2 & 14,3 & 29,4 & 29,2 & 0,16 \\
\hline aPL (\%) & 12,5 & 30,4 & 25 & 26,3 & 20,4 & 0,1415 \\
\hline
\end{tabular}


The aim ofthis study is to describe clinical and serologic characteristics of SLE patients according to complement levels. Methods Our SLE electronic database was analyzed (Jan 2014-Aug 2016). We included patients 18 years, fulfilling ACR 1997/SLICC 2012 classification criteria, with at least one year follow-up and at least two complement levels determinations at different times during follow-up.

Patients were classified in 4 groups, defined as following: Group 1 (normal C3 and C4 on all determinations), Group 2 (C3 and C4 below reference levels on all determinations), Group 3 (only $\mathrm{C} 4$ below reference levels on all determinations) and Group 4 (C3 and/or C4 on variable levels, with at least one determination below reference levels).

Demographic data, disease activity by SELENA-SLEDAI, accrual damage by SLICC-DI, presence of anti DNAds and anti $\mathrm{Sm}$ antibodies and clinical manifestations were assessed.

Statistical analysis was performed using Epi info v7. Correlation was assessed using chi2 or Fisher's test appropriate.

Results 149 patients were included. 95.3\% female, mean age at diagnosis was 30.6 years (CI: 28,5 32,8), mean disease duration 9.7 years. Mean SLICC-DI by group: Group 1 1.0, Group 2 0.5, Group 31.3 and Group 4 0.6.

All groups with hypocomplementaemia showed a higher SLEDAI respecting Group $1(\mathrm{p}=0.0013)$ (table 1)

Presence of anti DNAds and anti $\mathrm{Sm}$ antibodies is shown in table 1.

Respecting clinical manifestations, a significant difference was found in Lupus Nephritis (Group $136.8 \%$, Group 2 44.1\%, Group $378.6 \%$ and Group 4 36,4\% $(\mathrm{p}=0.0002)$ ) and hemolytic anemia (Group $328.6 \%$ and Group 1 17.6\% (p: 0.0196))(table 1).

Conclusions An association between C4 persistently below reference levels (Group 4) and anti DNAds antibodies and Lupus Nephritis was found. Group 3 patients may have a worse prognosis due to renal involvement.
Complement levels during follow up could be used as a marker to assess nephritis risk in SLE patients.

Funding Source(s): None

\section{SAFETY AND EFFICACY OF BELIMUMAB FOR TREATING SYSTEMIC LUPUS ERYTHEMATOSUS IN THE AFRICAN AMERICAN POPULATION AT LOUISIANA STATE UNIVERSITY HEALTH SCIENCES CENTER IN NEW ORLEANS}

Jose A Leon de la Rocha*, Emilie Bourgeois, Myriam Guevara. Louisiana State University Health Sciences Center New Orleans

\subsection{6/lupus-2019-Ism.156}

Background The efficacy of belimumab in African American (AA) patients has not been clearly proven. Manufacturer recommends use with caution in AA because efficacy in this population has not been well established. We aim to report clinical outcomes and safety of belimumab therapy in our AA population with Systemic Lupus Erythematosus (SLE) at Louisiana State University Health Sciences Center.

Methods This is a single center, descriptive, retrospective case series report. We used electronic medical records to identify AA patients, 18 years or older, diagnosed with seropositive SLE according to SLICC criteria, who had received belimumab in combination with standard therapies. Demographics, comorbidities, clinical outcomes, laboratory outcomes, medication utilization and adverse events are reported.

Results We identified 15 patients who started belimumab from March 2011 to March 2018. Only 5 met our study criteria. All patients were female with a mean age of 41.4 years at belimumab initiation (baseline). The average time since SLE diagnosis at baseline was 13.5 years. The average time on belimumab at the time of analysis was 19.06

\section{Abstract 156 Table 1}

\begin{tabular}{|c|c|c|c|c|c|c|c|c|c|c|}
\hline & \multicolumn{2}{|c|}{ Patient A } & \multicolumn{2}{|c|}{ Patient B } & \multicolumn{2}{|c|}{ Patient C } & \multicolumn{2}{|c|}{ Patient D } & \multicolumn{2}{|c|}{ Patient E } \\
\hline $\begin{array}{l}\text { Age when started } \\
\text { Belimumab (years) }\end{array}$ & \multicolumn{2}{|c|}{36.75} & \multicolumn{2}{|c|}{26.08} & \multicolumn{2}{|c|}{47.08} & \multicolumn{2}{|c|}{47.5} & \multicolumn{2}{|c|}{49.5} \\
\hline $\begin{array}{l}\text { Time of SLE diagnosis } \\
\text { (years) }\end{array}$ & \multicolumn{2}{|c|}{1} & \multicolumn{2}{|c|}{2} & \multicolumn{2}{|c|}{12} & \multicolumn{2}{|c|}{47.5} & \multicolumn{2}{|r|}{5} \\
\hline $\begin{array}{l}\text { Time on Belimumab at } \\
\text { of analysis (months) }\end{array}$ & \multicolumn{2}{|c|}{12.7} & \multicolumn{2}{|c|}{8.2} & \multicolumn{2}{|c|}{19} & \multicolumn{2}{|c|}{47} & \multicolumn{2}{|c|}{8.13} \\
\hline $\begin{array}{l}\text { Time on Belimumab } \\
\text { when reported benefit } \\
\text { (months) }\end{array}$ & \multicolumn{2}{|c|}{$<1$} & \multicolumn{2}{|c|}{$<1$} & \multicolumn{2}{|c|}{$<1$} & \multicolumn{2}{|c|}{6} & \multicolumn{2}{|r|}{5} \\
\hline $\begin{array}{l}\text { Dose of steroids at } \\
\text { baseline and analysis } \\
\text { (mg/day) }\end{array}$ & 40 & 0 & 20 & 10 & 10 & 10 & 10 & 10 & \multicolumn{2}{|c|}{ None } \\
\hline $\begin{array}{l}\text { Steroid Sparing Agent } \\
\text { at baseline and } \\
\text { analysis }\end{array}$ & $\mathrm{HCQ}$ & $\mathrm{HCQ}$ & None & $\mathrm{HCQ}$ & $\mathrm{HCQ}$ & $\mathrm{HCQ}$ & $\begin{array}{l}\mathrm{HCQ} \\
\text { MMF }\end{array}$ & $\begin{array}{l}\mathrm{HCQ} \\
\mathrm{MMF}\end{array}$ & $\begin{array}{c}\text { HCQ } \\
\text { MTX } \\
\text { MMF 1g }\end{array}$ & $\begin{array}{c}\text { HCQ } \\
\text { MTX } \\
\text { MMF 0.5g }\end{array}$ \\
\hline $\begin{array}{l}\text { Adverse Events of } \\
\text { Belimumab }\end{array}$ & \multicolumn{2}{|c|}{$\begin{array}{l}\text { Upper Respiratory } \\
\text { Infection, } \\
\text { Conjunctivitis }\end{array}$} & \multicolumn{2}{|c|}{$\begin{array}{l}\text { Flu - like } \\
\text { symptoms }\end{array}$} & \multicolumn{2}{|c|}{ Arthralgia } & \multicolumn{2}{|c|}{$\begin{array}{l}\text { Infusion reaction: } \\
\text { malaise, swollen } \\
\text { eyes, myalgia, } \\
\text { nausea and } \\
\text { vomiting }\end{array}$} & \multicolumn{2}{|c|}{ None } \\
\hline
\end{tabular}

